\title{
ESCOLHA DE UMA NOVA ÁREA PARA IMPLANTAÇÃO DE UM CENTRO DE CULTURA E LAZER EM PRESIDENTE PRUDENTE- SP
}

Jaqueline Naomi Haga, Mayara Pissutti Albano.

Universidade do Oeste Paulista - UNOESTE, Faculdade de Engenharias e Arquitetura e Urbanismo, Presidente Prudente, SP. E-mail: ma.albano@unoeste.br

\section{RESUMO}

O lazer e a cultura possuem inúmeras potencialidades, inclusive a de auxiliar na quebra das barreiras sociais, uma vez que o interesse por determinada atividade pode existir por indivíduos de diversas classes. No Brasil, o campo do lazer e da cultura ainda não é muito valorizado e se vê amparado em políticas públicas que pouco contribuem para o seu desenvolvimento, limitando o acesso a uma minoria da população brasileira. O presente trabalho busca analisar a oferta de equipamentos voltados a atividades de lazer e cultura na cidade de Presidente Prudente - SP e, a partir das análises realizar a escolha de um terreno adequado para implantação de um projeto que abrigue ambas as atividades e que atenda a população de classe média e baixa que tem o acesso a tais atividades dificultado. Para tanto, a metodologia adotada se baseia em pesquisas bibliográficas, documentais e de campo.

Palavras-chave: Lazer. Cultura. Centro de cultura. Centro de lazer. Presidente Prudente - SP

\section{CHOOSING A NEW AREA FOR IMPLANTATION OF CULTURAL AND LEISURE CENTER IN PRESIDENTE PRUDENTE - SP}

\begin{abstract}
The leisure and culture have great potential, including its aid in the breakdown of social barriers, as the interest in a particular activity may exist for individuals of different classes. In Brazil, the field of leisure and culture is not highly valued and it is seems to be supported by public policies that little contribute to their development, limiting the access to a minority of the population. This study seeks to analyze the supply of equipment geared to leisure activities and culture in the city of Presidente Prudente - SP, and from the analysis perform the choice of a suitable ground for the implementation of a project that houses both activities and which must attend lower and middle class population that has access to such activities hindered. Therefore, the methodology adopted is based on bibliographical research, documentary and field.
\end{abstract}

Keywords: Leisure. Culture. cultural center. Leisure center. Presidente Prudente - SP 


\section{INTRODUÇÃO}

Nas últimas décadas, especialmente após a Revolução Industrial, a forma com que as pessoas têm ocupado seu tempo livre, se tornou objeto de vários estudos adquirindo visibilidade. Mesmo sendo as atividades de lazer e cultura um direito de toda a população brasileira assegurado pela Constituição de 1988, a oferta de equipamentos e atividades voltadas para ambas as atividades nas cidades é bastante limitada, principalmente nos bairros mais carentes que dependem exclusivamente do setor público.

o que se observa é que, mesmo quando o poder público se manifesta e cria espaços destinados a tais atividades, o faz apenas pela exigência da implantação dos mesmos em função da legislação, sendo comum a presença de praças e quadras esportivas como equipamentos de lazer na maioria dos bairros, enquanto que equipamentos voltados à cultura quase que inexistem, o que resulta na construção de espaços que não conseguem atender as necessidades dos variados grupos de pessoas e seus interesses.

A presente pesquisa se origina da carência que muitos bairros na cidade de Presidente Prudente possuem de equipamento voltados para o lazer e cultura. Tem como objetivo a escolha e análise de uma área para implantação de um novo centro de cultura e lazer na zona noroeste da cidade, região composta em sua maioria por bairros de classe baixa e média, que tem o acesso dos moradores, aos poucos equipamentos voltados para tais atividades existentes na cidade, dificultado pela distância entre os bairros e os equipamentos mais relevantes que se encontram na porção oposta da cidade.

\section{METODOLOGIA}

A metodologia utilizada nesta pesquisa foi desenvolvida através da investigação qualitativa, utilizando-se de levantamento bibliográfico, de pesquisa documental, de levantamentos físicos arquitetônicos in locu na zona noroeste da referida cidade.

Primeiramente foi realizado levantamento bibliográfico em livros e periódicos com as principais obras referentes aos assuntos trabalhados, bem como pesquisas documentais pertinentes. Num terceiro momento foram realizados levantamentos in locu, que serviram de base para as análises do terreno escolhido. Após a conclusão das fases iniciais foram desenvolvidas as análises do referido lote, escolhido para inserção do centro de cultura e lazer.

\section{CENTROS DE LAZER E CULTURA}

Definir o conceito de centro cultural é tarefa difícil. O próprio significado da palavra cultura, que está ligada ao termo de forma bastante direta, ainda gera discussões entre os estudiosos do tema. A definição de centro cultural, pode ser dada porém, "pelo seu uso, e atividades nele desenvolvidas."(NEVES, 2013, p. 2).

Um espaço que difunda, além de conhecimento e informações relacionadas a cultura, diversas atividades que atuem de "[...]maneiras interdependentes, simultâneas e multidisciplinares[...]" (NEVES, 2013, P.3), de forma que integre os diferentes grupos de classes sociais. Segundo Neves $(2013$, p. 3) "[...] quem entra num centro cultural deve viver experiências significativas e rever a si próprio e suas relações com os demais".

Neves (2013) apresenta os verbos informar, discutir e criar, que juntos auxiliam na compreensão da finalidade e funcionamento de um centro cultural, e pondera que:

As atividades relacionadas ao verbo informar são desenvolvidas em espaços como teatros de arena, bibliotecas, cinemas, espaços de exposições, e outros ambientes semelhantes funcionalmente. O segundo, discutir, é viabilizado em ambientes como auditórios, salas de vídeo conferências, e até mesmo em espaços de convivência social, salas de 
reuniões e de múltiplo uso, pátios, dentre outros. Já o verbo criar são necessários espaços como ateliês de produção de obras novas, restauração, ou ainda de ensino para profissionais e aprendizes, mais conhecidas como oficinas de arte (NEVES, 2013, p. 5).

Da mesma forma, definir o que é lazer, é tarefa composta da mesma complexidade, e ainda bastante discutida entre os estudiosos. "A própria origem da palavra lazer patenteia esta variedade de opções: vem do latim licere, ser permitido, isto é, ser lícito escolher a maneira de aproveitar o tempo disponível". (MEDEIROS, 1975, p. 4). Já segundo o Novo Dicionário Aurélio, o significado de lazer é dado como ócio, descanso, folgar, vagar; não sendo difícil relacionar lazer à ócio (MARCELLINO, 2000).

Para Medeiros (1975, p. 3) o lazer pode ser conceituado “[...]como aquele espaço de tempo não comprometido, do qual podemos dispor livremente, porque já cumprimos nossas obrigações de trabalho e de vida." Para Gaelzer (1985, p. 48) o lazer implica em nada mais do que, a habilidade de cada um de ser ele mesmo, ou seja, o seu eu:

[...] lazer significa estar engajado em alguma atividade livremente, sem compulsão de forças externas ou direções internas neuróticas. Envolve espontaneidade de ação, liberdade de ser ele mesmo, acompanhado por uma áurea prazerosa, ausência de pressão e relax. Engajar-se na atividade porque encontra satisfação nela e realizá-la por encontrar aperfeiçoamento daquilo que é meta final.

Visto como uma forma de aliviar as tensões da mente, Medeiros (1975), também considera o lazer como uma válvula de segurança, uma ferramenta de bem-estar público, uma vez que quando utilizado de maneira construtiva, dá um sentido e finalidade ao tempo livre.

Marcellino (2000, p. 39), cita o trabalho de Dumazedier (s.d.) onde este "[...]distingue cinco categorias quanto ao conteúdo das atividades de lazer: os interesses físicos, os práticos ou manuais, os artísticos, os intelectuais, e os sociais." Ainda segundo o autor, independente da categoria de escolha da atividade a ser praticada, é preciso equilíbrio.

Não é difícil compreender que a camada da população com maior poder aquisitivo tem certos privilégios no que diz respeito ao lazer, com maior tempo disponível e condições para as atividades relacionadas ao mesmo (MARCELLINO, 2000). Porém, isto não significa que a população de classe social mais baixa desconheça a existência e importância do lazer (GAELZER, 1985). É comum a reivindicação por áreas de lazer e de equipamentos, em especial da população de bairros mais distantes das áreas centrais quando estas são consultadas em reportagens e programas de prestação de serviços (MARCELLINO, 2000).

Para Marcellino (2000), a distância entre moradia e equipamento, somada a um sistema de transporte coletivo precário, e as barreiras sócio-econômicas, somente contribuem para que grande parte da população se confine à práticas conformistas e, somente com a democratização do espaço é que se alcança um lazer também democratizado. "[...]não há como fugir do fato: o espaço para o lazer é o espaço urbano." (MARCELLINO, 2000, p.57).

O lazer, assim como a cultura podem ser considerados então, como uma ferramenta que quando bem utilizada e apresentada ao público faz com que este vivencie a ruptura das barreiras sociais que se resultam quando ambas oferecem estruturas e pessoas capacitadas para tal.

\section{CENTROS DE CULTURA E LAZER EM PRESIDENTE PRUDENTE - SP}

$\mathrm{Na}$ cidade de Presidente Prudente, localizada no i oeste do estado de São Paulo, à $558 \mathrm{~km}$ da capital paulista, possui aproximadamente 207600 habitantes (IBGE, 2010). Apesar da Lei de Parcelamento, Uso e Ocupação do Solo Urbano (Lei Federal no 6.766/79) afirmar que áreas 
destinadas ao lazer se façam presentes em loteamentos, e do Plano Diretor Municipal (Lei Complementar no 151/2008) defender a importância da cultura para a população, estes não trazem especificações relativas à qualidade tanto das atividades desenvolvidas quanto dos equipamentos necessários para as mesmas.

Quando se observa os espaços que o poder público destina ao lazer e à cultura, o que se encontra, na maioria dos bairros, voltados para o lazer são pequenas praças que contam com parquinho para crianças e academia da terceira idade, ou, áreas maiores que possuem geralmente quadra de esportes, pista de caminhada; enquanto no campo cultural, com exceção das iniciativas e projetos realizados no Centro Cultural Matarazzo, praticamente inexistem.

Hoje, as principais atividades voltadas para a cultura acontecem no Centro Cultural Matarazzo, enquanto que as atividades voltadas ao lazer são, em sua maioria, desenvolvidas no Parque do Povo e SESC (Figura 1). Os três espaços se destacam na cidade na oferta de lazer e cultura para a população, apesar de não serem facilmente acessíveis à todos.

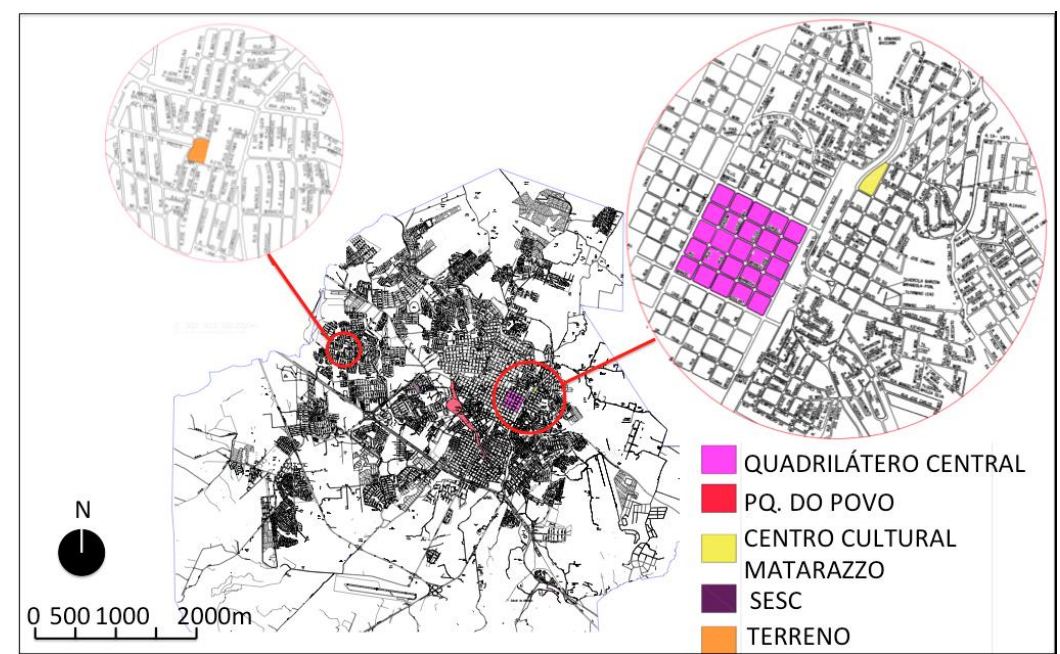

Figura 01. Mapa da cidade e seus principais equipamentos de lazer e cultura. Fonte: Prefeitura de Presidente Prudente, 2015. Editado: HAGA, 2016.

Com um crescimento da malha urbana da cidade significativo a noroeste, resultante da grande quantidade de loteamentos destinados à população de baixa renda na mesma, ainda assim, os equipamentos urbanos existentes deixam muito a desejar e a população que reside nestes bairros tem então que se deslocar até os espaços anteriormente citados para que possa desenvolver e gozar das atividades de lazer e cultura, e como muitos dependem de transporte público, a carência na qualidade do mesmo faz com o trajeto se torne cansativo e estressante, o que pode levar a muitos, a desistir das atividades de lazer em espaços externos.

No lazer, o que se observa nestes bairros é a presença de inúmeras áreas verdes deterioradas, destinadas a tais atividades das quais muitas, se encontram em estado decadente pela falta de manutenção onde as atividades oferecidas são sempre as mesmas, enquanto que as atividades culturais nem mesmo em um espaço de baixa qualidade são desenvolvidas.

A criação de um centro de lazer e cultura nestes bairros, além dos benefícios já citados que o lazer e a cultura podem proporcionar, proporcionará um espaço com o qual a população e os moradores de áreas mais próximas passam a se identificar. Para tal, a escolha da área para a um futuro projeto arquitetônico, se deu com o objetivo de oferecer atividades de lazer e cultura para a parcela da população que reside a noroeste da cidade, região que inicialmente se caracterizava por bairros destinados à população de baixa renda, hoje já conta também com uma população de classe média baixa e média.

Segundo dados do último censo do Instituto Brasileiro de Geografia e estatística (IBGE) de 2010, que realizam suas pesquisas na cidade através da definição de setores, aproximadamente 
dez mil moradores, de um total de 10 (dez) setores que se encontram mais próximos da área no qual o projeto será proposto, podem ser beneficiados com o projeto.

O considerável número de bairros que podem ser beneficiados com o projeto na área proposta, vai de acordo com o que defende Marcellino (2000 , p. 76) que "o importante num primeiro momento, é contribuir para que o maior número de pessoas tome parte nas atividades, atendendo a seus interesses de lazer", e partir desde primeiro contato, lhes apresentar um outro universo de atividades de lazer e cultura.

\section{TERRENO ESCOLHIDO}

O terreno escolhido está localizado em uma gleba destinada pela prefeitura para fins de lazer, que segundo a Lei de Parcelamento do Solo da cidade (Lei no 154/2008), deve contar com porcentagem entre $10 \%$ (dez por cento) a 15\% (quinze por cento) do total do loteamento.

Diferente do que ocorre em muitos bairros nos quais, as áreas destinadas ao lazer são inseridas em sobras de terrenos localizados nos fundos dos bairros, este, se encontra em ponto privilegiado no mesmo, logo no início do bairro, com acesso e vista facilitados pelas avenidas próximas, totalizando quase trinta mil metros quadrados, que são separados em quatro lotes. Dos quatro terrenos destinados ao lazer, o terreno escolhido para a implantação do projeto possui uma área de 3.926,85 $\mathrm{m}^{2}$ (Figura 2).

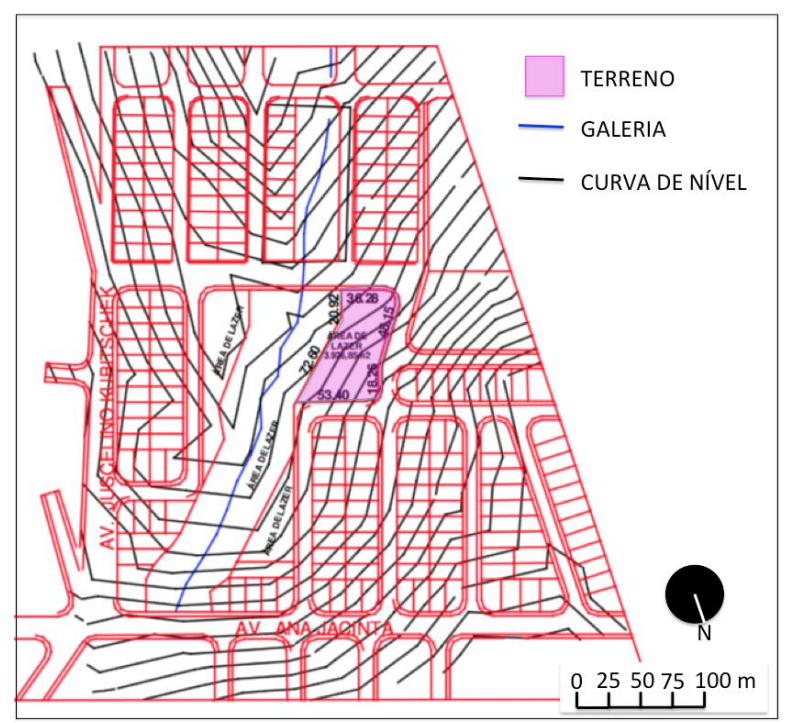

Figura 02. Terreno com curva de nível e galeria.

Fonte: Prefeitura de Presidente Prudente, 2015. Editado: HAGA, 2016.

O terreno conta com desnível acentuado de quase seis metros, desnível este que, pode ser observado mais facilmente quando não se está dentro do próprio terreno. A área de lazer é cortada por uma galeria (figura 3), e apesar da distância entre o terreno e a galeria ser superior ao mínimo exigido pelo novo código florestal (quinze metros), atenção especial deverá se dada no momento em que o equipamento urbano for construído.

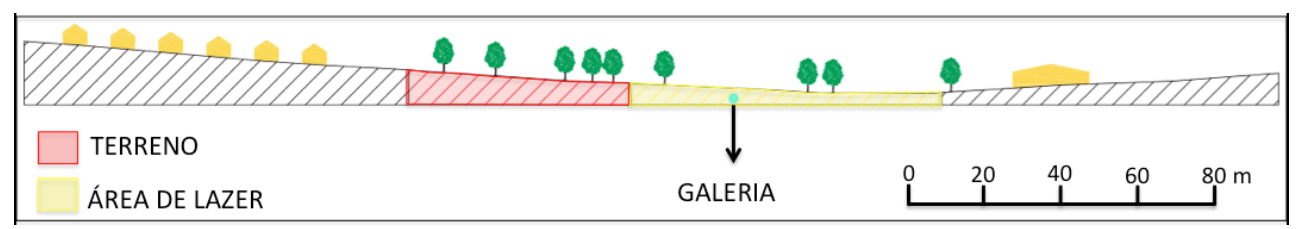

Figura 03. Corte

Fonte: HAGA, 2016. 
Uma vez que a intenção do projeto é oferecer lazer não só para a população dos bairros próximos ao terreno, mas também para qualquer pessoa que tiver interesse em usufruir do espaço, a presença de duas vias arteriais responsáveis pela ligação dos bairros é mais um ponto positivo para a escolha da área. As seguintes vias são responsáveis por esta ligação: Avenida Ana Jacinta e Avenida Pres. Juscelino Kubitschek de Oliveira (Figura 4). A primeira, é importante principalmente por ser a única avenida no local a receber o fluxo de automóveis que vem da área central, passando pelo shopping center Prudenshopping, seguindo em direção a Cohab; já a segunda, pode tanto ser um meio para se chegar à área a partir dos bairros que se situam entre o terreno e a rodovia Raposo Tavares, e também é opção de rota escolhida para aqueles que saem da cidade pela mesma rodovia.

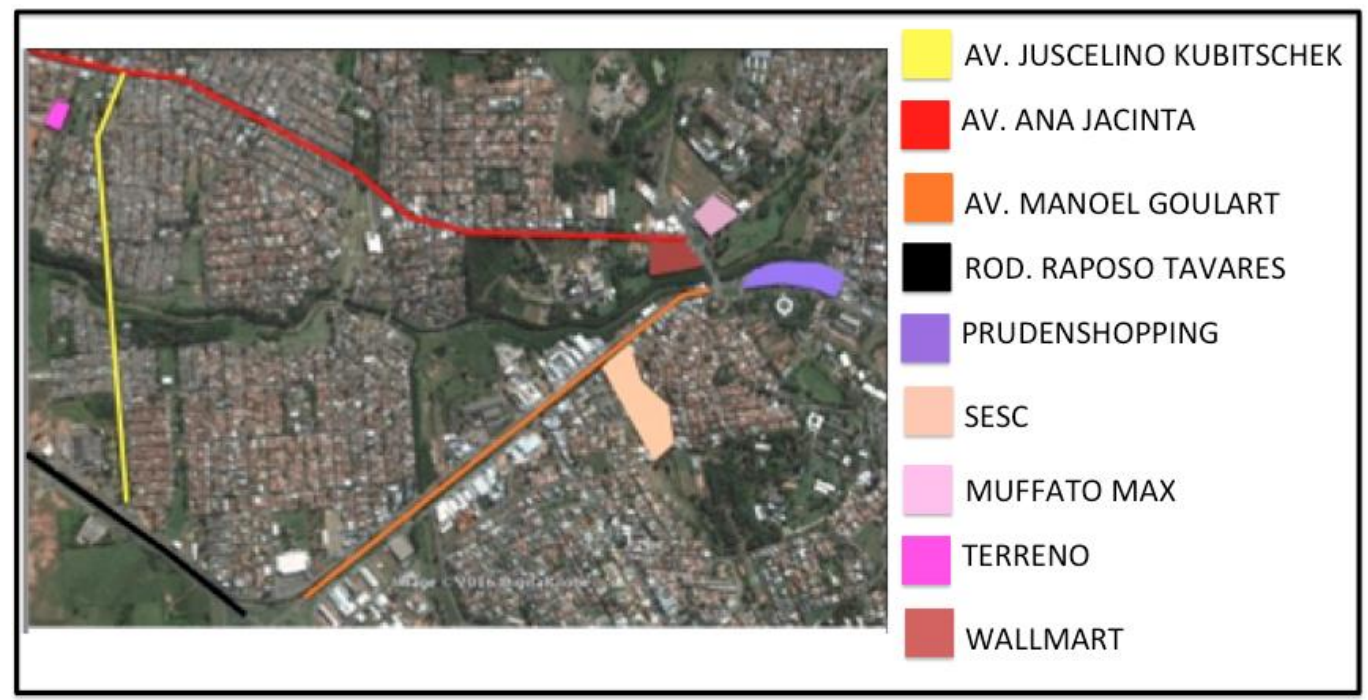

Figura 04. Acessos principais

Fonte: Google Maps, 2016. Editado: HAGA, 2016.

O projeto que deverá ser proposto posteriormente no lote escolhido para tal deverá tem como objetivo principal oferecer atividades de lazer e cultura para a população residente nos bairros a leste e noroeste da cidade, de forma que a interação que resulta dos diferentes grupos de pessoas e seus interesses, fortaleça os laços sociais entre os usuários do Centro. Para isso o projeto precisará ser um espaço aberto e convidativo ao público, chamando a atenção da população para esta porção da cidade através deste novo atrativo de lazer e cultura, e o terreno escolhido permite que o projeto cumpra este propósito, em função das características supracitadas, como dimensões suficientes, fácil acesso e localização estratégica.

\section{CONCLUSÃO}

Fica claro a importância que a temática relacionada ao lazer e a cultura tem para o desenvolvimento social e do indivíduo, e da necessidade da oferta de espaços de qualidade e pessoal capacitado no auxilio das atividades oferecidas nos mesmos.

A viabilidade da área escolhida para a implantação de um centro de cultura e lazer se dá pois, quando instalado, deverá atender um público que hoje tem seu acesso ao lazer e a cultura limitado pelas condicionantes existentes na cidade de Presidente Prudente - SP e auxiliará na ampliação do acesso de camadas sociais mais baixas a esse tipo de atividade em um espaço de qualidade.

\section{REFERÊNCIAS}

BRASIL. Congresso Nacional. Lei n. 6.766 de 19 de dezembro de 1979. Dispõe sobre o parcelamento do solo e dá outras providencias. 
BRASIL. Congresso Nacional. Constituição Federal, 1988.

GAELZER, L. Ensaio à liberdade: uma introdução ao estudo da educação para o tempo livre. 1. ed. Porto Alegre: D. C. Luzzatto, 1985.

MARCELLINO, N. C. Lazer e educação. 6. ed. Campinas: Papirus, 2000.

MEDEIROS, E. B. O lazer no planejamento urbano 2. ed. Rio de Janeiro: Fundação Getúlio Vargas, 1975.

NEVES, R. R. Centro cultural: a cultura à promoção da arquitetura. Centro Cultural a Cultura à promoção da Arquitetura, Goiânia, v. 1, n. 5, jul., 2013. Disponível em: < http://www.ipog.edu.br/revista-especialize-online-busca/?autor=Renata\%20Ribeiro\%20Neves>. Acesso em: 20 mar. 2016.

PRESIDENTE PRUDENTE. Prefeitura Municipal. Lei Complementar 151/2008 de 10 de janeiro de 2008. Dispõe sobre a Lei do Plano Diretor do município de Presidente Prudente. Disponível em: < http://www.presidenteprudente.sp.gov.br/site/Documento.do?cod=606> Acesso em: 5 abr. 2016.

Prefeitura Municipal. Lei Complementar 154/2008 de 10 de janeiro de 2008. Dispõe sobre a Lei de Parcelamento do Solo para fins urbanos e dá outras providências. Disponível em: < http://www.presidenteprudente.sp.gov.br/site/Documento.do?cod=614> Acesso em: 9 abr. 2016. 